\title{
Lineardirektantrieb für Transportbänder
}

\author{
Dipl.-Ing. Mišel Radosavac ${ }^{1)}$, Dipl.-Ing. Tobias Froböse ${ }^{1)}$, \\ Prof. Dr.-Ing. Ludger Overmeyer ${ }^{1)}$ \\ Dipl.-Ing. Jan-Peter Jastrzembski ${ }^{2)}$, Prof. Dr.-Ing. Bernd Ponick ${ }^{2)}$ \\ 1) Institut für Transport- und Automatisierungstechnik, Leibniz Universität Hannover \\ ${ }^{2)}$ Institut für Antriebssysteme und Leistungselektronik, Leibniz Universität Hannover
}

Abstract: In der Intralogistik erfolgt der Antrieb von Transportbändern bei vielen Anwendungsszenarien durch eine oder mehrere Antriebstrommeln am Kopf bzw. Ende der Förderstrecke. Die gesamte Vorschubkraft wird dabei von der Antriebstrommel auf das Transportband übertragen. In diesem Bereich wird das Transportband besonders beansprucht. Das Institut für Transport- und Automatisierungstechnik (ITA) hat zusammen mit dem Institut für Antriebs- und Leistungselektronik (IAL) der Leibniz Universität Hannover ein neues Antriebskonzept auf Basis eines Linearmotors realisiert. Mit diesem Konzept lassen sich der Antrieb und damit die Krafteinleitung gleichmäßig über die Förderstrecke verteilen. Dadurch wird das Förderband geringer beansprucht als bei Systemen mit angetriebenen Trommeln. Anhand von Messungen im laufenden Betrieb wird der Versuchsförderer hinsichtlich der effektiv nutzbaren Vorschubkraft, dem Wirkungsgrad, dem Reibungsverhalten und dem Temperaturverlauf in der Antriebskomponente untersucht. Die Ergebnisse tragen zur konstruktiven Optimierung bei und können als Entscheidungsgrundlage für die Fördersystemauswahl berücksichtigt werden.

\section{$1 \quad$ Einleitung}

Förderbänder werden in der Intralogistik für den Transport von Stückgütern sowie leichten Schüttgütern eingesetzt. Der Transportbandantrieb erfolgt dabei größtenteils über eine am Kopf oder am Ende des Transportbands befindliche Antriebstrommel, welche über ein Getriebe mit einem Motor verbunden ist. Eine zweite Trommel sorgt für die Umlenkung und die Bandvorspannung. Um eine Masse bewegen zu können, wird die notwendige Kraft über die Antriebstrommel in das Transportband eingeleitet. In Verbindung mit den zu befördernden Lasten wird das Band in diesem Bereich mit hohen Zugkräften beaufschlagt, denen der Aufbau des Förderbands standhalten muss. Die zulässige Zugkraft bestimmt weiterhin die maximale Bandlänge. Sollen größere Strecken überwunden werden, ist entweder ein stärkeres Band oder die Verteilung auf mehrere hintereinander liegende Förderstrecken erforderlich.

Lineardirektantriebe übertragen Vorschubkräfte ausschließlich über das elektromagnetische Feld und damit kontaktlos. Aufgrund der fehlenden mechanischen Übertragungselemente tritt keine zeitliche Veränderung der Betriebseigenschaften auf. Durch die Möglichkeit, die Krafteinleitung entlang des Transportbands verteilen zu können, wird das Transportband geringer beansprucht 
als bei Konzepten mit angetriebenen Trommeln. Dadurch lässt sich das Förderband hinsichtlich der aufzunehmenden Zugkräfte deutlich einfacher und damit kostensparender dimensionieren. Die Verwendung von Zwischenantrieben auf Basis eines Lineardirektantriebs erlaubt zudem die Möglichkeit die Krafteinleitung genau steuern zu können, wodurch sich diese Antriebsart zum Positionieren von Gütern auf dem Transportband eignet. Daraus ergeben sich Anwendungen zum Beispiel für Quergurtsorter. Ebenso kann durch die verteilte Krafteinleitung das Laufverhalten der zum Haftgleiteffekt (stick-slip-effect) neigenden Werkerbänder verbessert werden.

\section{Versuchsförderer}

Das Institut für Transport- und Automatisierungstechnik (ITA) hat zusammen mit dem Institut für Antriebs- und Leistungselektronik (IAL) der Leibniz Universität Hannover einen Versuchsförderer entwickelt, bei dem ein Transportband als aktive Antriebskomponente durch einen Linearmotor direkt angetrieben wird.

Abbildung 1 zeigt den Aufbau des Versuchsförderers, der sich im Wesentlichen aus einem Transportband, welches als Kunststoffmodulkettenband ausgeführt ist, dem Lineardirektantrieb (Schnittansicht) und dem Steuerungsschrank zusammensetzt. Mit Hilfe des eingebauten Gleichstrommotors lässt sich im motorischen Betrieb eine bestimmte Last erzeugen, die vom Transportband überwunden werden muss. Dadurch können unterschiedliche Belastungsszenarien nachgestellt werden. Im generatorischen Betrieb wird die durch den Lineardirektantrieb an das Transportband abgegebene Leistung messen. Aus der Differenz zwischen abgegebener und aufgenommener Leistung sind Rückschlüsse auf Verluste wie z.B. durch Reibungsbeiträge möglich. Die Schnittansicht in Abbildung 1 zeigt die Anbindung des Lineardirektantriebs im Versuchsförderer. Die Antriebskomponente setzt sich aus dem Primär- und Sekundärteil zusammen. Während der Primärteil des Linearantriebs (Ständer) stationär am Rahmen angebracht ist, sind die Sekundärteile des Linearantriebs (Läuferelemente) direkt auf dem Kunststoffmodulband befestigt.

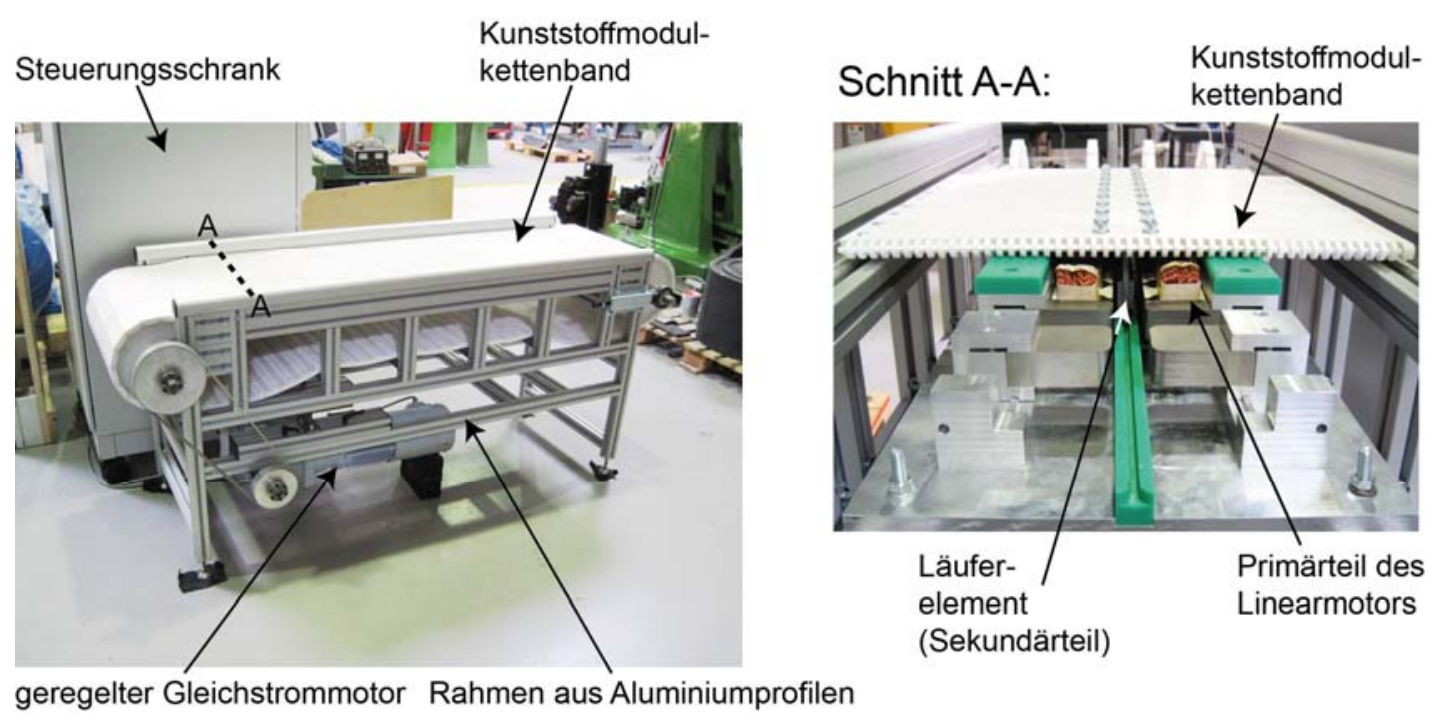

Abbildung 1: Versuchsförderer 
Die vom Lineardirektantrieb erzeugte Vorschubkraft wird folglich über die Läuferelemente direkt auf das Kunststoffmodulband übertragen.

Im Folgenden werden die Antriebskomponente und die Rolle des Transportbands für den Antrieb näher erläutert.

\subsection{Antrieb}

Ausgehend von unterschiedlichen Antriebstopologien ([All76], [Bud83]) liegt der Fokus im Rahmen des vorliegenden Versuchsförderers auf Synchron-Antrieben. Der wesentliche Vorteil derartiger Antriebe besteht darin, dass auf stromführende Leiter im Sekundärteil (bewegter Läufer) verzichtet werden kann und folglich weder eine Stromzuführung (z.B. über Schleifkontakte) notwendig ist noch ein zusätzlicher Wärmeeintrag in das Förderband stattfindet.

Durch die Forderungen nach einem kompakten Motor für den Transportbandantrieb, einer hohen Vorschubkraft und der konstruktiven Umsetzbarkeit in Hinblick auf die Luftspalttoleranzen zwischen Primär- und Sekundärteil wird hier das Konzept der Permanentmagneterregten Synchronmaschine (PMSM) für den Transportbandantrieb verwendet (siehe [Ove09]). Im bewegten Sekundärteil (Läufer) der PMSM werden Magnete angeordnet. Abhängig von der Leistungsfähigkeit und dem Einsatzgebiet können dabei unterschiedliche Magnetwerkstoffe verwendet werden. Die Läuferelemente sind mit dem Transportband verbunden. Der unbewegte Primärteil (Ständer) besteht aus einer mehrsträngigen Wicklung. In Wechselwirkung mit einem vom bestromten Ständer generierten Wanderfeld ( $\mathrm{vgl}$. Drehfeld), wird eine nutzbare Vorschubkraft erzeugt [Fis09], wodurch sich das Transportband antreiben lässt.

Im Wesentlichen soll der Lineardirektantrieb im Versuchsförderer folgende Anforderungen erfüllen:

- frei wählbare Vorschubgeschwindigkeiten im Bereich von $0 \mathrm{~m} / \mathrm{s}$ bis $2 \mathrm{~m} / \mathrm{s}$

- Vorschubkraft von ca. $200 \mathrm{~N}$

- kompakter Aufbau

- Erweiterbarkeit durch Hinzufügen weiterer Linearantriebe

\subsection{Transportband}

Als Transportband werden Kunststoffmodulbänder eingesetzt, da der Versuchsförderer flexibel gestaltet sein soll, um beispielsweise den Austausch der Läuferelemente im Nachhinein zu gewährleisten. Die Kunststoffmodulbänder weisen gegenüber flexiblen Förderbändern nur eine geringe Dehnung auf. Somit ist ein konstanter Abstand der Läuferelemente, und damit der Polteilung, gewährleistet. Durch die Möglichkeit das Kunststoffmodulband an einer beliebigen Stelle zu öffnen, wird zudem die Montage und Demontage des Transportbands vereinfacht. 
Die Läuferelemente sind durch Schraubverbindungen mit den Kunststoffmodulbandelementen verbunden. Abbildung 2 (links) zeigt den dazugehörigen Aufbau. Im Einzelnen setzen sich die Läuferelemente aus jeweils einem Trägerelement, der sogenannten Stahlfahne, und den darauf befestigten Magneten zusammen. Während dem Betrieb tauchen die Läuferelemente in den Motor ein, die jeweils von beiden Seiten mit Statorhälften umschlossen werden. Abbildung 2 (rechts) zeigt den verwendeten doppelseitigen Aufbau.
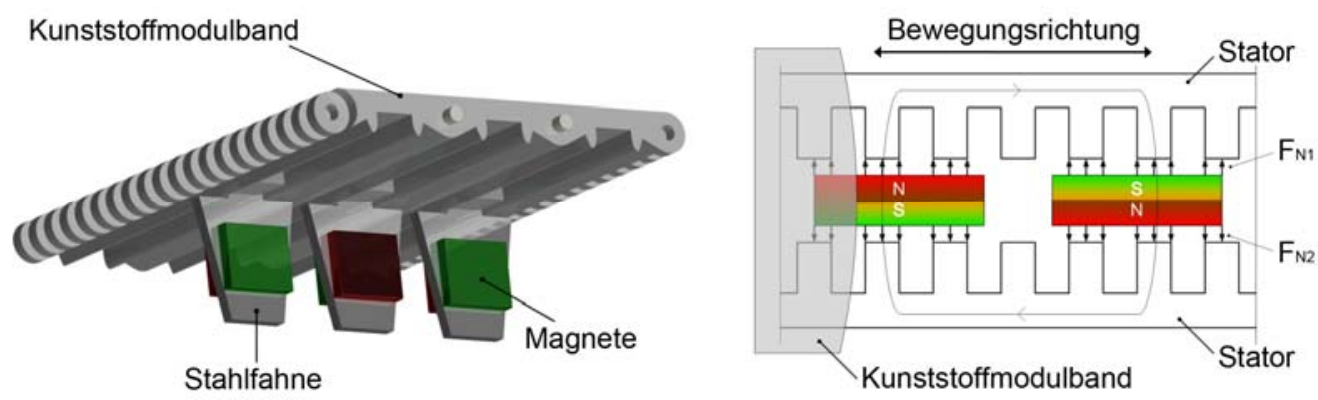

Abbildung 2: Förderbandmodell mit Läuferlementen (links) und Ausführungsform der Stator-I Läuferanordnung (rechts)

Zwischen dem Stator und den Läuferelementen entstehen prinzipbedingt Normalkräfte. Diese wirken im Allgemeinen anziehend und können bei einer einseitigen Statoranordnung Größenordnungen von ca. dem acht- bis zwölffachen der Vorschubkraft erreichen, wodurch unerwünschte Reibungsbeiträge entstehen können. Durch den doppelseitigen Aufbau im Versuchsförderer entsteht zwischen Stator und Läufer auf jeder Seite eine Normalkraft $F_{N}$. Bei einer idealen, mittigen Ausrichtung des Läuferelements sind die Beträge der beiden Normalkräfte $F_{\mathrm{N} 1}$ und $\mathrm{F}_{\mathrm{N} 2}$ gleich (Abbildung 2, rechts). Durch die umgekehrten Vorzeichen beträgt die Summe beider Normalkräfte Null. Das System befindet sich im Gleichgewicht. Abweichungen aus der mittigen Lage führen jedoch zu ungleichen Beträgen der Normalkräfte $F_{\mathrm{N} 1}$ und $F_{\mathrm{N} 2}$, wodurch das Läuferelement $z \mathrm{u}$ einer Seite angezogen wird. Im ungünstigen Fall entsteht ein Kontakt zwischen der Läufer- und der Statorfläche. Dies führt zu Reibungsbeiträgen während der Transportbandbewegung.

Die Verwendung von Permanentmagneten erzeugt zu jedem Zeitpunkt einen magnetischen Fluss. Folglich kann der Motor in Abhängigkeit von der Läuferlage auch Kräfte im unbestromten Zustand entwickeln. Die Rastkräfte treten in Permanenterregten Maschinen an allen Stellen in Erscheinung, an denen unterschiedlich große Luftspalte zwischen Läufer und Stator vorhanden sind. Aufgrund des nachteiligen Einflusses der Rastkräfte auf den Verlauf der Vorschubkraft, sind diese bereits beim Entwurf des Motors durch Geometrieanpassungen des Ständerblechpakets zu minimieren [Ste05]. Im vorliegenden Versuchsförderer konnten übliche Methoden zur Rastkraftminimierung (z.B. Schrägung) aus konstruktiven Gründen nicht angewendet werden. Aus FiniteElemente-Simulationen wurde eine Lösung entwickelt, bei der mittels einer zusätzlich eingeführten Quelle von Rastkräften eine Minimierung erreicht wird. Durch die 
Verwendung eines zusätzlichen und vom restlichen Stator getrennten Zahns, der einen gleichartigen Rastkraftverlauf wie der Stator erzeugt, jedoch gegenüber diesem verschoben ist, kompensieren sich die anteiligen Rastkräfte [Ove09].

\section{$3 \quad$ Messungen und Ergebnisse}

Ziel der Messungen ist die Überprüfung und Bewertung des Versuchsförderers hinsichtlich der in Abschnitt 2.1 beschriebenen Anforderungen. Im Fokus der Betrachtungen liegen insbesondere die effektiv nutzbare Vorschubkraft, die Reibungsverluste und das Temperaturverhalten in Abhängigkeit von der Betriebszeit. Der Versuchsförderer wird zu diesem Zweck um entsprechende Messapparaturen erweitert und das Betriebsverhalten aufgezeichnet. Dadurch sollen Rückschlüsse auf den Wirkungsgrad sowie das Langzeitverhalten ermöglicht werden. Die daraus gewonnenen Ergebnisse sollen zur konstruktiven Optimierung des Versuchsförderers sowie als Entscheidungsgrundlage für die Fördersystemauswahl beitragen.

\subsection{Reibungsverluste}

Reibungsverluste können im Versuchsförderer in folgenden Bereichen auftreten:

- Kunststoffmodulband/Tischauflage Mit zunehmendem Transportbandgewicht steigen die Reibungsverluste zwischen dem Förderband und der Tischauflage.

- Läuferelemente/Führungsschiene

Mit der Führungsschiene werden die Läuferelemente auf Abstand zum Ständerblechpaket gehalten (siehe Abbildung 3). Aufgrund von konstruktionsbedingten Toleranzen kann die ideale, mittige Ausrichtung der Läuferelemente abweichen. Dadurch können unerwünschte Normalkräfte entstehen, die den Bewegungsfluss stören und folglich die Reibung erhöhen. Damit ein Verkanten der Läuferelemente bei der Führungsschiene vermieden wird, sind die Ein- und Auslaufbereiche abgerundet.

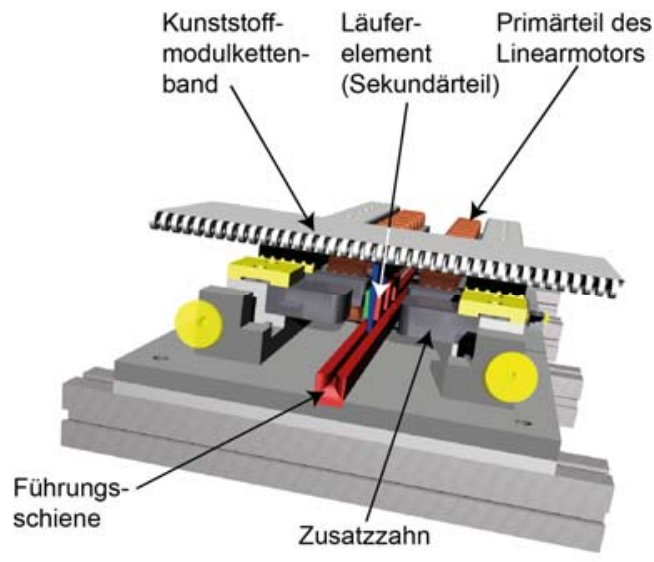

Abbildung 3: Linearmotor mit Förderband 
Die entstehende Reibung zwischen den einzelnen Kunststoffmodulbändern ist unabhängig vom Antriebskonzept und wird daher im Rahmen dieser Untersuchungen nicht näher betrachtet.

Für die Bestimmung der auftretenden Reibungsbeiträge wird das Förderband mittels des in Abbildung 1 gezeigten Gleichstrommotors angetrieben. Die dabei aufgewendete Kraft wird zusammen mit der Transportbandgeschwindigkeit in eine äquivalente Leistung umgerechnet. Diese Leistung ist erforderlich, um das Transportband bei einer bestimmten Geschwindigkeit zu bewegen. Da die Reibung ein Teil der aufgewendeten Gesamtleistung ist, wird der Versuchsförderer mit drei verschiedenen Konfigurationen unter zwei konstanten Förderbandgeschwindigkeiten näher betrachtet:

1. Der Gleichstrommotor treibt das Förderband ohne die Läuferelemente und ohne den Linearmotor an.

2. Der Gleichstrommotor treibt das Förderband mit den Läuferelementen ohne den Linearmotor an.

3. Der Gleichstrommotor treibt das Förderband mit Läuferelementen und eingebautem Linearmotor an.

Die erste Konfiguration entspricht einem Standardaufbau eines in der Intralogistik verwendeten Förderers. Mit der zweiten Konfiguration und der daraus entstehenden Leistungsdifferenz zur ersten Konfiguration, lässt sich der Einfluss des Gewichts der Läuferelemente auf die entstehende Reibung untersuchen. Durch die dritte Konfiguration können Rückschlüsse auf den Reibungsanteil zwischen den Läuferelementen und der Führung geschlossen werden. Die experimentell gewonnen Ergebnisse aller Konfigurationen zeigt Abbildung 4:

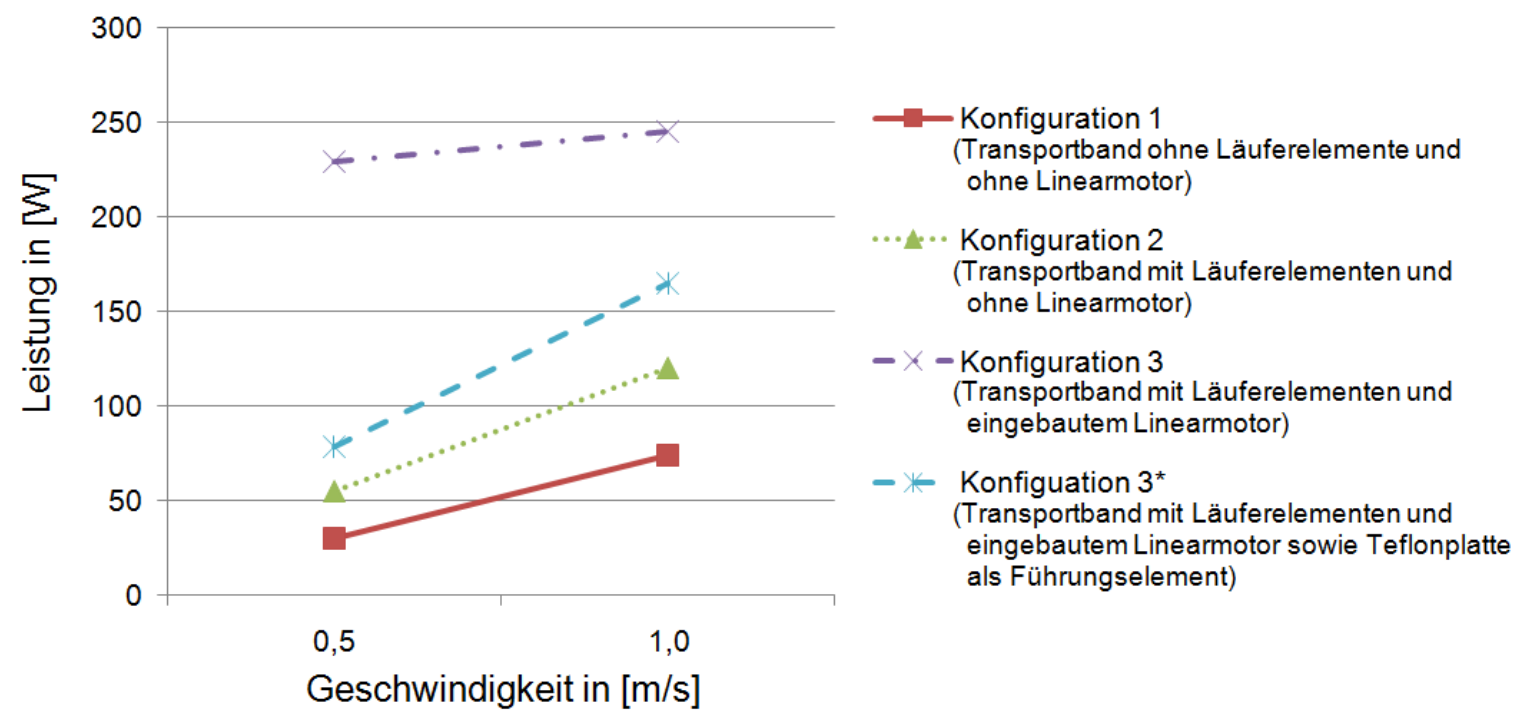

Abbildung 4: Aufgewendete Leistung des Gleichstrommotors bei unterschiedlichen Transportbandgeschwindigkeiten 
Im Gegensatz zu Konfiguration 1, bei welcher das Förderband ohne die Läuferelemente und den Linearmotor angetrieben wird, ist eine lineare Zunahme des Leistungsbedarfs durch das Hinzufügen der Läuferelemente bei Konfiguration 2 deutlich ersichtlich. Die benötigte Leistung zur Kompensation der Reibungsverluste infolge der Gewichtserhöhung liegt im Mittel bei ca. $35 \mathrm{~W}$. Bei eingebautem Linearmotor steigt der Leistungsbedarf bei Konfiguration 3 gegenüber Konfiguration 2 und einer Bandgeschwindigkeit von 0,5 m/s um $174 \mathrm{~W}$ an. Demzufolge hat die Reibung im Bereich der Führungsschiene einen um ca. Faktor fünf höheren Anteil an der Zunahme des Leistungsbedarfs gegenüber der Gewichtszunahme der Läuferelemente. Die Zunahme des Leistungsbedarfs bei Konfiguration 3 gegenüber Konfiguration 2 liegt bei einer Bandgeschwindigkeit von $1,0 \mathrm{~m} / \mathrm{s}$ bei $125 \mathrm{~W}$. Demnach sinkt insgesamt der negative Einfluss der Führungsschiene mit zunehmender Bandgeschwindigkeit.

Aufgrund des hohen Einflusses der Führungsschiene an der Gesamtreibung wurde diese durch eine Teflonfolie ersetzt. In Abbildung 5 ist die Teflonfolie als weißer Streifen erkennbar. Die eingesetzte Teflonfolie ist $1 \mathrm{~mm}$ stark und damit nur geringfügig dünner als der erforderliche Luftspalt. Die Folie liegt zudem an den luftspaltseitigen Flächen der Statorhälften auf. Da Teflon schwierig zu verkleben ist, wird die Platte lediglich an den Stirnseiten mit einer Schraube gegen Verschieben in Längsrichtung gesichert. Durch die Teflonfolie wird gewährleistet, dass die Läuferelemente nicht verkippen können und außerdem der geforderte Luftspalt eingehalten werden kann. Sowohl in den Betriebseigenschaften, als auch in der Handhabung bei der Montage, hat sich diese Art der Führung als besonders einfach herausgestellt. Den benötigten Leistungsbedarf für die Bewegung des Transportbands mit Verwendung der Teflonfolie zeigt Konfiguration $3^{*}$ in Abbildung 4. Die Zunahme des Leistungsbedarfs bei eingebautem Linearmotor und der Teflonfolie steigt im Mittel gegenüber Konfiguration 2 um ca. 35 W an. Damit liegt der Reibungseinfluss durch Gewichtszunahme der Läuferelemente in einer ähnlichen Größenordnung wie bei den Verlusten bei der Reibpaarung der Läuferelemente und der Teflonfolie.

\subsection{Rastkräfte}

Die Rastkraftmessung hat das Ziel die auftretenden Rastkräfte unter Betriebsbedingungen zu erfassen. Der Rastkraftverlauf wird dabei unter dem Einfluss von Reibung im statischen Zustand gemessen, da die Umsetzung des Messaufbaus aufgrund der reibungsbehafteten Führung der Messapparatur vom idealisierten Finite-Elemente-Modell abweicht.

Abbildung 5 zeigt den Aufbau der Messapparatur. Für die Messung werden 18 Läuferelemente mit Magneten an einem Aluminiumprofil befestigt, das mit Hilfe einer Kugelspindel durch den Linearstator gefahren wird. Die Kugelspindel ist über eine Kraftmessdose mit dem Gestell des Versuchsförderers verbunden und wird von einem Schrittmotor angetrieben. Die Kraftmessdose ist so angeordnet, dass die 
auftretenden Kräfte in Verfahrrichtung als Messsignal zur Verfügung gestellt werden. Der Schrittmotor ist über eine Faltenbalgkupplung mit der Kugelspindel gekoppelt, so dass dieser nahezu keine Kräfte in axialer Richtung aufnehmen kann. Der Verfahrweg des Aluträgers kann über den Schrittwinkel und der Steigung der Spindel errechnet werden. Der Träger zieht sich durch das Auftreten von Normalkräften in Richtung einer Statorhälfte.

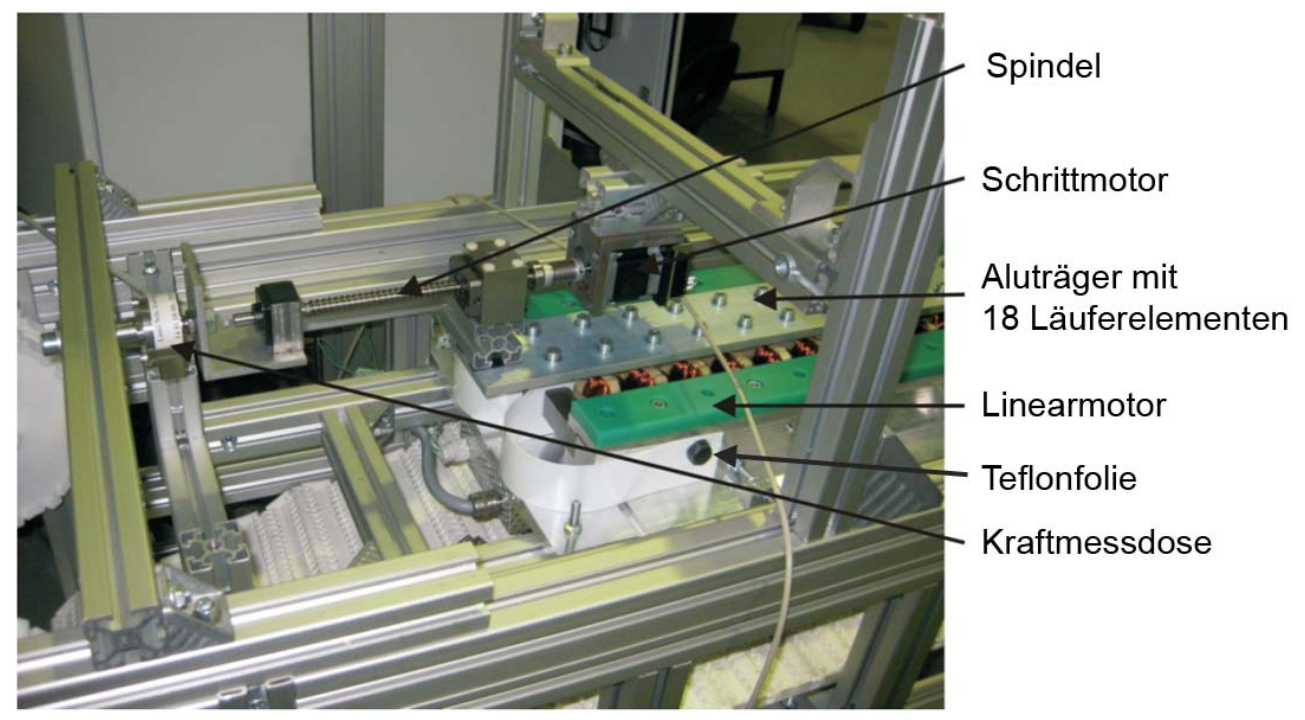

Abbildung 5: Messaufbau zur Rastkraftmessung

Als Gleitfläche wird die Teflonfolie jeweils auf der luftspaltseitigen Statorfläche angeordnet. Der Aluträger wird allein durch das Auftreten der magnetischen Kräfte in der horizontalen Blechpaketmitte gehalten, weswegen auf weitere reibungsbehafteten Führungselemente verzichtet werden kann.

Abbildung 6 zeigt die errechneten Kraftverläufe und gemessenen Rastkraftverläufe. Dabei ist deutlich zu erkennen, dass die aufgezeichneten Kraftverläufe nicht mit den berechneten Rastkraftverläufen übereinstimmen. Dies kann durch die Abweichung der Läuferelemente aus der mittigen Lage und den damit verbundenen, hohen Normalkräften begründet werden.
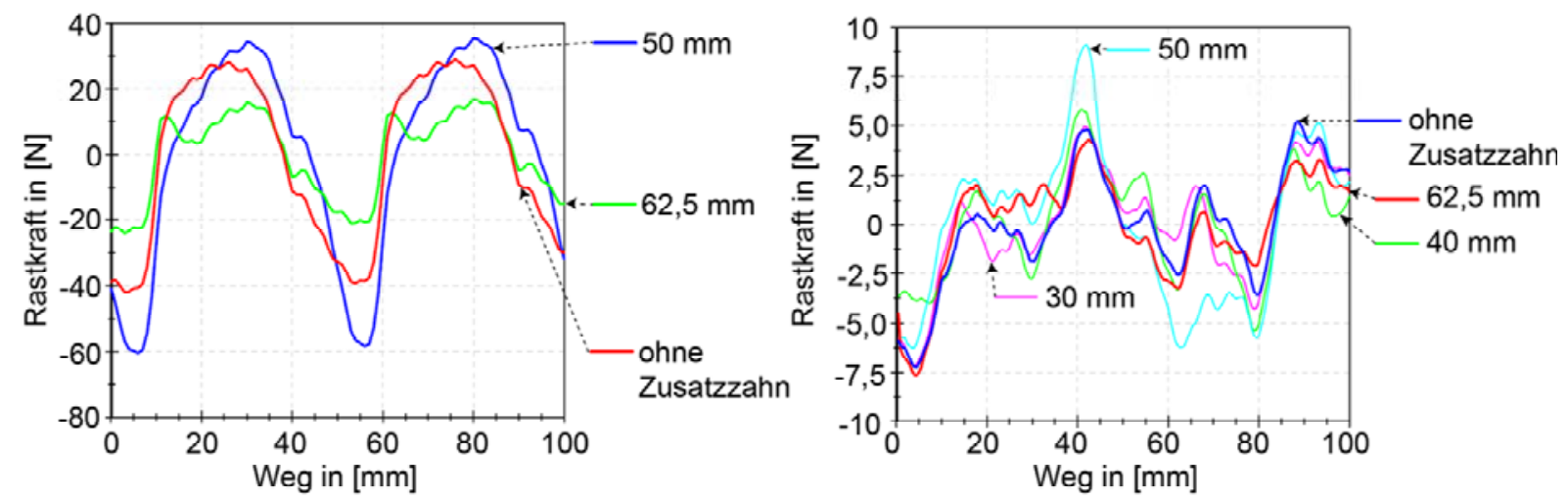

Abbildung 6: Errechnete (links) und gemessene (rechts) Rastkraftverläufe bei unterschiedlichen Abständen des Zusatzzahns 
Abbildung 7 zeigt die rechnerisch ermittelte Normalkraft in Abhängigkeit des Luftspalts:

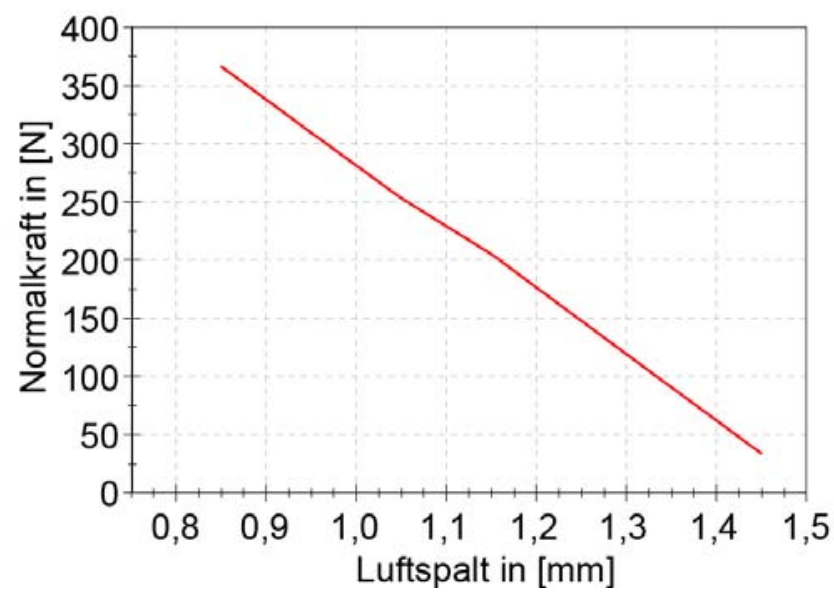

Abbildung 7: Normalkraft bei Auslenkung des Läufers aus der Mittellage

Verkleinert sich einer der Luftspalte, wodurch der Läufer nicht mehr in der Mittellage ist, steigt die Normalkraft stark an. Die Teflonfolie weist eine Sollstärke von $1 \mathrm{~mm}$ bei einer Toleranz von $\pm 0,2 \mathrm{~mm}$ auf. Somit können Normalkräfte von bis zu $400 \mathrm{~N}$ auftreten. Bei einem theoretischen Reibkoeffizienten von Stahl zu Teflon von 0,04 entstehen Reibkräfte von ca. $16 \mathrm{~N}$. Dies gilt sowohl für das Haften als auch für das Gleiten. Daher können Rastkräfte, die unterhalb dieser Reibkraft liegen, nicht gemessen werden. Lediglich die hohen Rastkraftspitzen können einen Einfluss auf das System ausüben. Demzufolge können die Rechenergebnisse aus Abbildung 6 mit dieser Art der Messung nur tendenziell verglichen werden, da die Reibung den Verlauf stark verfälscht. Desweiteren ist erkennbar, dass der Zusatzzahn einen Einfluss auf den Rastkraftverlauf hat. Der errechnete optimale Abstand von $62,5 \mathrm{~mm}$ führt wie gewünscht auf eine minimale Kraftschwankung. Durch die Reibung aufgrund der Normalkraft wird der Rastkraftverlauf gedämpft. Folglich ist der Zusatzzahn nicht notwendig. Bei den Messergebnissen in Abbildung 6 ohne Zusatzzahn, wirkt sich die Reibung sogar positiv auf die Kraftschwankungen aus, da die gemessenen Amplitudenwerte klein sind.

\subsection{Verlustleistungen und Temperaturverlauf}

Im Linearmotor entstehen während des Betriebs Verlustleistungen in Form von Kupfer- und Eisenverlusten. Die Eisenverluste können als vergleichsweise klein angenommen werden, da die speisende Frequenz von $7 \mathrm{~Hz}$ bei einer Vorschubgeschwindigkeit von $0,7 \mathrm{~m} / \mathrm{s}$ gering ist. Dominant treten daher die Kupferverluste hervor, die vom Widerstand der Statorwicklung und dem Strom abhängen. Durch die Erwärmung des Motors während des Betriebs, steigen der Widerstand der Statorwicklung und die Kupferverluste. Über die Beziehung

$$
R(\theta)=R\left(\theta_{0}\right) \cdot\left(1+\alpha_{C u^{\prime}}\left(\theta-\theta_{0}\right)\right)
$$


und der Kenntnis des Temperaturkoeffizienten $\alpha_{\mathrm{Cu}}$ von Kupfer sowie des Kaltwiderstands $R\left(\theta_{0}\right)$, kann der Warmwiderstand $R(\theta)$ der Wicklung für beliebige Temperaturunterschiede zwischen der Raumtemperatur $\theta_{0}$ und der Wicklungstemperatur $\theta$ errechnet werden.

Der Motor wird zur Aufnahme der Temperaturverläufe aus einem Frequenzumrichter gespeist, der gegenwärtig noch nicht mit einer Positionserfassung der Läuferelemente ausgerüstet ist. Für die Aufnahme der Temperaturverläufe wird der Motor folglich ungeregelt betrieben. Aus Sicht der Kraftentwicklung lässt sich unter dieser Bedingung kein optimaler Betriebspunkt einstellen. Dennoch lassen sich am Frequenzumrichter die Ausgangsfrequenz und der Strom einstellen. Dadurch kann das thermische Verhalten abgebildet und durch Messung der elektrischen Größen die abgegebene Leistung bestimmt werden. Zur Messung des Temperaturverhaltens befinden sich je Statorhälfte drei Sensoren in den Wickelköpfen auf der dem Transportband abgewandten Seite, sowie ein Sensor in der Rückseite des Motorträgers. Die Temperaturverläufe in Abbildung 8 (links) zeigen die Temperaturen an den acht Positionen innerhalb des Linearmotors.

Die Differenzen zwischen den Temperaturkurven einzelner Sensoren weisen auf eine unterschiedliche thermische Anbindung an die Wicklung. Der Motor wurde in allen drei Strängen $U, V$ und $W$ mit einem identischen Strom betrieben, weshalb auch von etwa gleichen Temperaturverhältnissen auszugehen ist. Die höchste Temperatur verzeichnet der Sensor in Strang $U$, weswegen dieser als Maßgabe für die Stromeinstellung verwendet wurde.
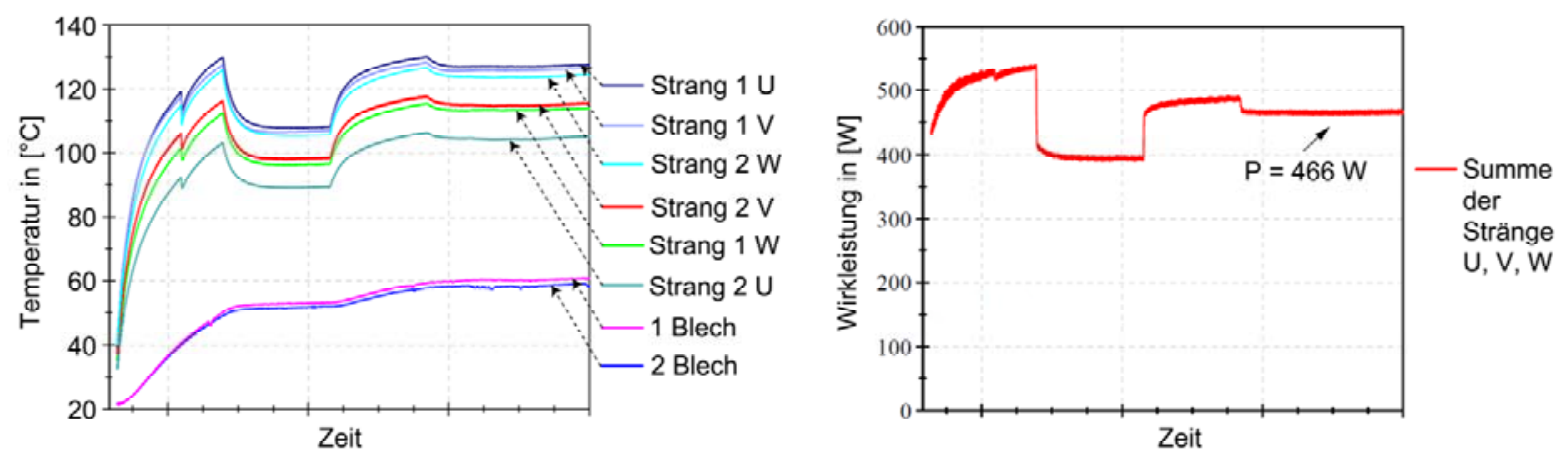

Abbildung 8: Temperaturverläufe (links) und Wirkleistungsverlauf (rechts)

Für den durchgeführten Temperaturverlauf sollte die Wicklungstemperatur einen Maximalwert von $130{ }^{\circ} \mathrm{C}$ nicht überschreiten. Im Kaltzustand beträgt der Ständerwiderstand des Motors 29,4 $\Omega$ und der lineare Temperaturkoeffizient von Kupfer beträgt $0,0039 \mathrm{~K}^{-1}$. Der Warmwiderstand bei einer Wicklungstemperatur von $130{ }^{\circ} \mathrm{C}$ und einer Raumtemperatur von $20^{\circ} \mathrm{C}$ errechnet sich demnach zu $42 \Omega$.

Um die gewünschte Temperatur einzuhalten, wurde der Strom mehrfach angepasst, weswegen die Verläufe in Abbildung 8 (links) Sprünge aufweisen. Bei einem Strom von 1,66 A erreicht die Wicklungstemperatur schließlich einen Endwert von $127^{\circ} \mathrm{C}$. Die Gesamtdauer der Temperaturverlaufsmessung betrug 7 Stunden, wobei sich die (c) 2011 Logistics Journal : Proceedings - ISSN 2192-9084 
Temperatur in der letzten Stunde vor dem Abschalten um weniger als $1^{\circ} \mathrm{C}$ verändert hat. Der Motor hat demzufolge den thermischen Beharrungszustand erreicht.

Während der Temperaturverlaufsmessungen hat der Linearmotor den abgeschalteten Gleichstromgetriebemotor mit angetrieben. Durch die Aufzeichnung der anliegenden Kraft sowie der Drehzahl konnte auf die Leistung zum Antreiben des Gleichstrommotors zurückgerechnet werden. Abbildung 8 (rechts) zeigt, dass der Motor insgesamt eine Wirkleistung von $466 \mathrm{~W}$ im thermischen Beharrungszustand aufnimmt. Der gemessene Warmwiderstand führt zusammen mit dem Strom in Höhe von 1,66 A zu einem Kupferverlustanteil von 347 W. Die Differenz von 119 W entfällt auf die restlichen Beiträge. Die am Getriebe gemessene Leistung betrug laut Messungen $25 \mathrm{~W}$. Durch Reibung fallen nach den vorherigen Messungen rund $79 \mathrm{~W}$ an Verlusten an. Der Rest in Höhe von $15 \mathrm{~W}$ muss demnach die Eisenverluste decken. Abbildung 9 zeigt die prozentuale Aufteilung der einzelnen Leistungsanteile.

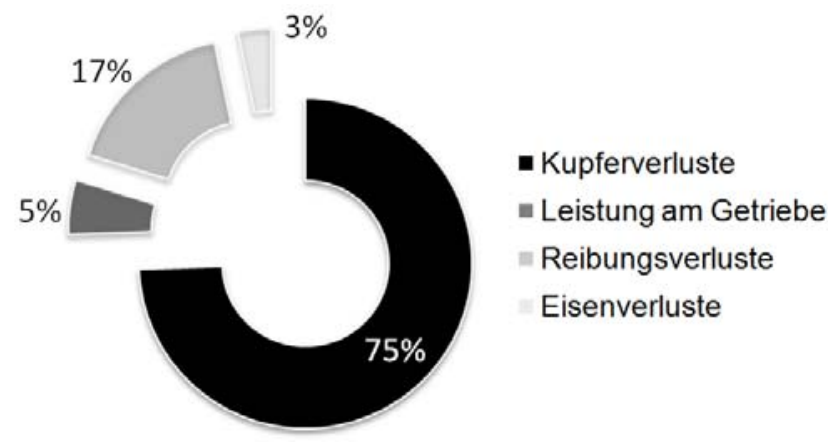

Abbildung 9: Leistungsanteile des Linearantriebs im Versuchsförderer

\subsection{Wirkungsgrad}

Aus den ermittelten Reibungs-, den Kupfer- und den Eisenverlusten sowie der mechanisch abgegebenen Leistung am Gleichstromgetriebemotor lässt sich die vom Linearmotor auf das Transportband wirkende Vorschubkraft berechnen. Bei einer Wirkleistung von $466 \mathrm{~W}$ im thermischen Beharrungszustand muss der Linearmotor insgesamt $79 \mathrm{~W}$ für die Deckung der Reibungsverluste und $25 \mathrm{~W}$ für die Bewegung des Transportbands im Versuchsförderer abgeben. Bei einer Vorschubgeschwindigkeit von $0,5 \mathrm{~m} / \mathrm{s}$ und einer abgegebenen Leistung von $104 \mathrm{~W}$ ergibt sich eine umgerechnete Vorschubkraft von $208 \mathrm{~N}$. Der Wirkungsgrad $\eta$ ist definiert als das Verhältnis der mechanisch abgegebenen Leistung (104 W) zur zugeführten Leistung (466 W). Folglich beträgt der Wirkungsgrad des betrachteten Linearmotors $22 \%$.

Der verwendete Linearmotor weist gegenwärtig einen Leistungsfaktor von $\cos \varphi$ mit 0,55 auf, da der Frequenzumrichter keine Positionserfassung der Läuferelemente aufweist. Bei einer positionsrückgeführten Regelung des Linearmotors wird der Leistungsfaktor und damit der Wirkungsgrad größer. Rein rechnerisch ergibt sich unter Berücksichtigung des thermisch noch zulässigen Stroms von ca. 1,7 A eine 
Vorschubkraft von 410 N. Gegenüber der angestrebten Vorschubkraft von 200 N besitzt der Entwurf folglich noch ausreichendes Potential zur Leistungssteigerung.

\section{$4 \quad$ Zusammenfassung und Ausblick}

Mit dem vorgestellten Konzept ist es erstmals gelungen, ein System zu entwickeln, bei dem ein Transportband als aktive Antriebskomponente durch einen Linearmotor direkt angetrieben wird. Das Antriebskonzept stell eine neue Anwendungsmöglichkeit in der Intralogistik dar, da sich die maximal auftretenden Zugkräfte im Transportband verringern lassen. Dadurch können längere Förderstrecken mit mehreren Motoren ohne Übergabe an weitere Transportbandsysteme umgesetzt werden.

Im vorliegenden Versuchsförderer wurden Kunststoffmodulbänder eingesetzt, die eine geringe Dehnung aufweisen und die einfache Austauschbarkeit von Läuferelementen ermöglichen. Im Rahmen der Ermittlung der Reibungsbeträge ließ sich der negative Einfluss der Führungsschiene identifizieren, welche daraufhin durch eine Teflonfolie ersetzt wurde. Die Messergebnisse dazu bestätigen den positiven Einfluss der Teflonfolie auf die Reibung. Ebenfalls konnte der positive Einfluss des Zusatzzahns auf die Rastkräfte validiert werden, allerdings wird aufgrund der auftretenden Reibung, die den Rastkraftverlauf dämpft, auf den Zusatzzahn verzichtet. Während der Temperaturverlaufsmessungen wurden zum einen der thermische Beharrungszustand des Motors und die Leistungen durch Kupfer- und Eisenverluste ermittelt. Anschließend ließ sich durch Berücksichtigung aller Verluste die effektiv wirkende Vorschubkraft sowie der Wirkungsgrad des Linearmotors im Versuchsförderer bestimmen. Die Ergebnisse können für die Entscheidungsunterstützung bei der Wahl eines Fördersystems verwendet werden. Durch den Einsatz einer Positionsrückführung der Läuferelemente des Linearmotors ist mit einer Steigerung der Vorschubkraft und damit des Wirkungsgrads zu rechnen.

Für die zukünftige Entwicklung soll der Versuchsförderer weiter optimiert und ein geeignetes Anwendungsszenario ausgewählt und untersucht werden. Dabei steht insbesondere der praktische Betrieb im Vordergrund. Aufgrund der hohen Verbreitung von flexiblen Förderbändern in der Intralogistik ist darüber hinaus die Integration der Läuferelemente in dieser Art von Bändern näher zu betrachten.

\section{Literatur}

[All76] Alles, R.: Zum Zwischenantrieb von Gurtförderern mittels angetriebener Tragrollen und Linearmotoren. Institut für Fördertechnik, Technische Universität Hannover (1976)

[Bud83] Budig, P.-K.: Drehstromlinearmotoren. Hüthig 1983

[Fis09] Fischer, R.: Elektrische Maschinen. Hanser, 2009 
[Ove09] Overmeyer, L.; Froböse, T.; Ponick, B.; Jastrzembski, J.-P.: Modellierung eines Lineardirektantriebs für Transportbänder. 5. Fachkolloquium der WGTL, S. 83-94 (2009)

[Ste05] Steinbring, J.: Kraftwirkungen in permanentmagneterregten Maschinen. Fortschritt-Berichte VDI, Reihe 21, Nr. 370, 2005 